Ponteconvo, G. (1955). J. gen. Microbiol. 12, 330-331

\title{
The Impact of Genetics
}

\author{
By G. PONTECORVO \\ Department of Genetics, The University of Glasgow
}

So far there has been no impact of genetics on microbial systematics. There has been a very fruitful one, of course, on the systematics of higher organisms. What is unwisely called 'The New Systematics' is the result of the recent introduction of evolutionary and genetic approaches into a systematics already well developed in a large number of groups of animals and plants.

The impact of genetics and evolution on microbial classification is bound to come. It will start as soon as the systematics of one or more groups of micro-organisms is sufficiently advanced: my guess is that the yeasts, the coliform bacteria, and the filamentous ascomycetes are going to be the most likely victims.

Classical systematics is one in which Linnaeus's idea that there are as many species as were created in the beginning is unconsciously still the master. Intraspecific variation is resented, let alone variation in time: it is a nuisance which interferes with classification. The 'new' systematics accepts enthusiastically variation at both these levels, and even makes use of it in its deductions.

Before I go into this matter, let me again emphasize: until the description of the forms of a particular group of organisms is well advanced, there is no question of 'new' systematics. It is estimated that over $90 \%$ of the living species of birds have been identified. In certain families of mammals, Lepidoptera, flowering plants and ferns the 'old' systematics is almost as advanced as this. In all these groups the first step in classification ("A species is a systematic unit considered as a species by a competent systematist') could be followed by one based on biometric, ecologic, genetic and cytologic analysis. Perhaps in about half a dozen groups of higher organisms this four-prong attack has begun. In micro-organisms no group is ripe as yet.

Now the contribution of genetics to the systematics of higher organisms has been mainly the following. Every organism is different from every other one. Many of these differences are due to differences in hereditary determinants like the genes, whose mechanism of transmission is fully understood. Others are due to differences in other transmissible determinants, whose nature and mode of transmission is unknown. A third kind of difference is untransmissible, such as age and most effects of the environment.

A dynamic definition of a species is the aggregate of individuals which, directly or indirectly, can contribute over an indefinite number of generations to a common pool of hereditary determinants. For instance, a male rabbit cannot pool its genes directly with another male rabbit, but it can do so through its progeny. In the simplest situation, a species includes all the potentially interbreeding individuals, the descendants of which are also potentially interbreeding. 
The criterion for classifying an individual into a species is that of its potential contribution to, or its sharing in, the gene pool of that species. Because of the nature of the hereditary process, the transmissible variation which is expressed and detectable in any one population is always a very minor part of its potential variation. The relative proportions of the two vary, even between populations of one species, with the environment and with the 'genetic system'. The 'genetic system' includes the mating structure of populations (inbreeding, sex differentiation, etc.), the details of the hereditary processes, the way in which the gene pool is distributed throughout the species, etc. All this varies tremendously both within and between species. A knowledge of the genetic system makes sense of the otherwise impossible systematics of Rubus or Primula, to give two examples from plants, and man or Drosophila, to give two examples from animals.

In conclusion, there is great individual variation within a species and to distinguish individuals of one species from those of a closely related one we use the yardstick of gene flow versus gene isolation. I hasten to repeat that even in the systematics of higher organisms this criterion is barely beginning to be used, and that for a long time in many groups it will have to be used only as an inference.

Now as to micro-organisms. I repeat that the systematics of no group is as yet ripe for the 'impact'. Even in the best described groups the proportion of forms described is very small. There is still an enormous amount of useful work to be done of a purely descriptive type, followed by as rough and as practical a classification as 'old' systematists can devise.

When the next stage will be reached we shall have some most interesting special problems set by micro-organisms as to their genetic systems. Besides the standard processes of sexual reproduction the transmission of hereditary determinants in micro-organisms takes a variety of novel forms. Already several of them have been discovered in barely 10 years of work: $(a)$ particulate cytoplasmic inheritance, such as that of Kappa in Paramecium and probably of cytochrome oxydase in yeast; $(b)$ non-particulate cytoplasmic inheritance such as that of antigens in Paramecium; (c) transformation in pneumococci; $(d)$ 'transduction' in Escherichia coli and Salmonella spp., besides what seems to be a proper sexual cycle in Escherichia coli; $(e)$ heterokaryosis in filamentous fungi; $(f)$ the 'parasexual' cycle in filamentous fungi; (g) recombination in coli-phages and in various animal viruses.

Clearly, even though most micro-organisms may be asexual-and we should not be too sure about that-they can cope otherwise with the necessity for pooling hereditary determinants. Even if the discovery of new processes of transmission and recombination of hereditary determinants continued at the present rate this should not prevent the introduction into microbial 'new systematics' of some criterion involving the idea of common ancestry and potentially common posterity. This has been the main contribution of genetic and evolutionary study to systematics in higher organisms and its stimulus has been very powerful. I am confident that it will be equally fertile in microbial systematics, where the difficulty of rapid variation in time is so much more telling than in higher organisms. 